\title{
A Gaint Oral Teratoid Tumor Combined Natal Teeth in Pediatrics: A Case Report
}

\section{Mohammed J Aboud*}

Consultant pediatric surgery, pediatric surgery unit, the Maternity and Child Teaching Hospital, Al Diwaniya, Iraq

*Corresponding Author: Mohammed J Aboud, Consultant pediatric surgery, pediatric surgery unit, the Maternity and Child Teaching Hospital, Al Diwaniya, Iraq.

Received: August 29, 2019; Published: September 20, 2019

DOI: 10.31080/ASPE.2019.02.0151

\begin{abstract}
Teratomas are benign neoplasms composed of all the three germinal layers. They are composed of tissue elements foreign to the anatomic site in which it arises. Only approximate $1.6 \%$ of these tumors are found in the oral region. Pure address within the oral cavity and the tongue is very rare. We report a case of a giant oral teratoma combined natal teeth discovered at birth, which was managed with success and followed up for six months. A full-term male neonate was born by vaginal delivery with a congenital tumor protruding from the mouth, a large pedunculated mass, which prevented oral feeding. After 10 days of neonatal intensive care unit (NICU) admission, the mass was excised under general anesthesia from the hard palate and a local palatal flap repaired the mucosal defect. Histopathology confirmed teratoma. This case provides hope to all colleagues to improve the outcome through precise prenatal diagnosis, evaluation, and management roadmaps of potential cases of epignathus.
\end{abstract}

Keywords: Oral; Teratoid; Epiganthus; Natal; Pediatrics

\section{Introduction}

Teratomas are benign neoplasms composed of all the three germinal layers. They are composed of tissue elements foreign to the anatomic site in which it arises. Around $80 \%$ are located in the ovaries and sacral lesions, $7 \%$ are seen in the head and neck region, only approximate $1.6 \%$ of these tumors are found in the oral region [1]. Pure address within the oral cavity and the tongue is very rare. Solely few numbers of cases are reported within the literature to date [2]. Epignathus is a highly developed congenital teratoid tumor in the oral cavity. Its incidence ranges from 1 in 35,000 to 1 in 200,000 live births and it usually affects the posterior nasopharynx, sphenoid or hard palate and has a slight female predominance [3]. This type of tumor may cause multiple malformations and sequelae in the patients, particularly in the case where the tumor mass is between the palatine processes before the sixth week of gestation and grows markedly from the seventh to a ninth week [4]. It may prevent fusion of the nasal septum with the bilateral palatine processes, which results in cleft palate formation. The tu- mor mass may result in morbidity and mortality due to their virtue of the location resulting in airway obstruction and respiratory distress [5]. Thus, the treatment of this tumor is complex and requires multiple interventions by a multidisciplinary team [6]. In 1950, authors introduced the terms 'natal teeth' for teeth present at birth and 'neonatal teeth' for teeth that erupt within the first 30 days of life. The incidence of natal and neonatal teeth has been submitted in multiple studies [7].

We report a case of a giant oral teratoma combined natal teeth discovered at birth, which was managed with success and followed up for six months.

Ethics statement

Written informed consent was obtained from the patients' parent who participated and managed in this report for publication of both cases and any accompanying images. The scientific and health committee in our Health directorate office approved this publication. 


\section{Case report}

A full-term male neonate was born by vaginal delivery at midwife, he is the product of a full-term pregnancy from a 19 year-old prim gravida mother. The pregnancy and delivery were uncomplicated and the intrapartum ultrasound examinations were normal. At birth, the weight was $2.660 \mathrm{~kg}$, with a congenital tumor protruding from the mouth, a large pedunculated mass, which prevented oral feeding. Surprisingly we noticed loose teeth in the lower front region of the jaw [Figure 1, A-C]. Over the following days, the mass was noticed to increase in size and by the 2nd day, the infant showed severe respiratory distress. He was referred to our hospital for consultation; a nasotracheal tube and an oral feeding tube were inserted initially. Oral clinical examination revealed a well-defined mass measuring $8 \times 6 \times 4 \mathrm{~cm}$, protruding from the oral cavity without adhesion to the oral vestibule, obliterating the buccal vestibule. It was soft in consistency and readily bled on touch, originating from the hard palate. The patient did not have a cleft palate. He was unable to close his mouth; his tongue was swollen, protruding, and displaced toward the palate. The ventral surface of the tongue was edematous. No communication between the tumor and the intracranial cavity could be detected. We recommended outpatient ears, nose, and throat follow-up, as there were no accompanying symptoms. Initial laboratory findings revealed a white blood cell count of $12.300 / \mathrm{mm} 3$, hemoglobin of $13.4 \mathrm{~g} / \mathrm{dL}$, erythrocyte sedimentation rate was $15 \mathrm{~mm} / \mathrm{hr}$. Serum electrolyte and blood urea nitrogen values are normal. Liver function tests were normal. Serum alpha-fetoprotein (AFP) was highly elevated (5600 KIU/L), B-human chorionic gonadotropin (B-HCG) was normal. Head, neck and paranasal sinus computerized tomographic (CT)-scan native study demonstrated an oropharyngeal soft tissue tumor 16,6 mm X 14,6 mm with fat density extended from upper oral cavity, attached to the hard palate, more on the right side, the bone intact with no erosion, the pharynx and larynx with normal boundaries. Opacification of the right sinuses by the fluid density with opacification of 3 - 4 teeth in the lower front region of the jaw [figure 2, A-C]. The nasal septum deviated to the right with non-patent right osteo meatal complex with no intracranial extension. The pediatric dentist carried his opinion that there was severe mobility (Grade II) associated with these teeth. A danger of aspiration of these teeth existed. A decision to extract them immediately was made after the prophylactic administration of vitamin $K$. The procedure was carried out on $3^{\text {rd }}$ day of admission under local anesthesia and careful curettage of the sockets was performed in an attempt to remove any odontogenic cellular remnants that might otherwise have been left in the extraction site. After 10 days of neonatal intensive care unit (NICU) admission, the mass was excised under general anesthesia from the hard palate and a local palatal flap repaired the mucosal defect. The histopathologic study revealed a mass composed of mature glial tissue, a wide range of cellular differentiation, a disorganized combination of mature adipose tissue, mucin secreting glands, tooth structure, skin annexes, skeletal muscle, and bone. No immature component was present. There was no evidence of malignancy. The diagnosis of teratoma was submitted [figure 3, A, B]. The postoperative course was uneventful. The nasotracheal and the gastric tube removed on the six post-operative days. Masseteric function and swallowing slowly improved over 2 - 3 weeks. The infant was discharged after 3 weeks, and he was symptom-free at 6 months follow-up.

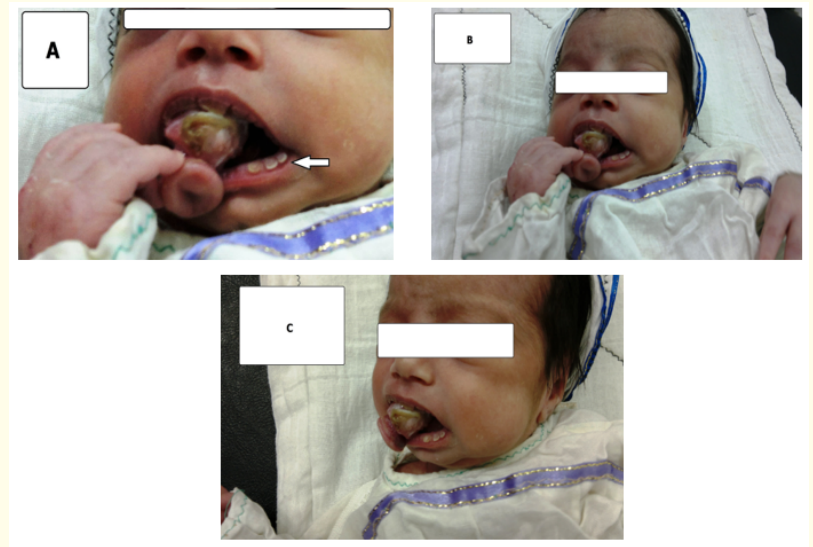

Figure 1 A-C: A well-defined mass protruding from the oral cavity without adhesion to the oral vestibule, obliterating the buccal vestibule with loose teeth in the lower front region of the jaw

(arrowed).

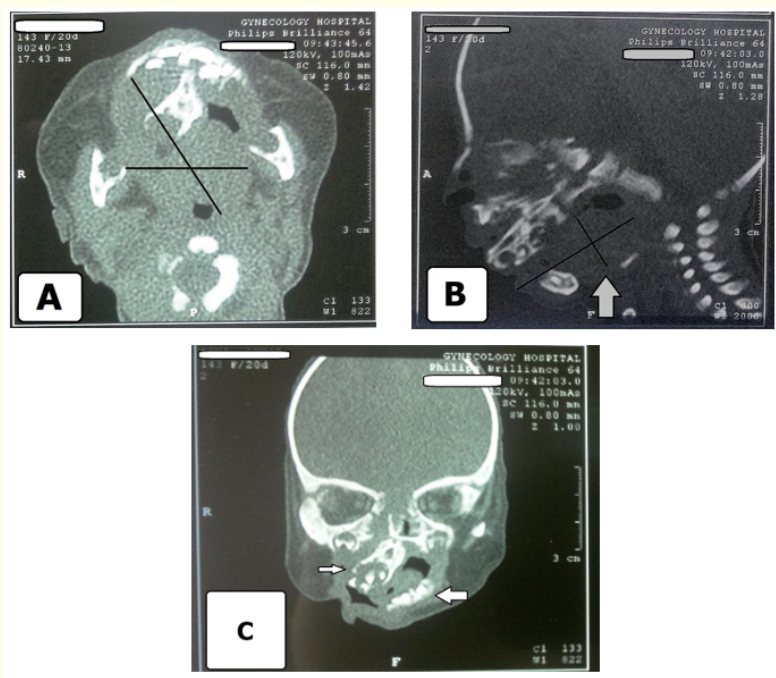

Figure 2 A-C: Native CT Images revealed A, oropharyngeal soft tissue tumor with fat density extended from the upper oral cavity, attached to the hard palate, more on the right side, the bone intact with no erosion, the pharynx and larynx with normal boundaries.

$B$ and C, opacification of the right sinuses by the fluid density with opacification of 3-4 teeth in the lower front region of the jaw (arrowed). The nasal septum deviated to the right (arrowed) with non-patent right osteo meatal complex with no intracranial extension. 

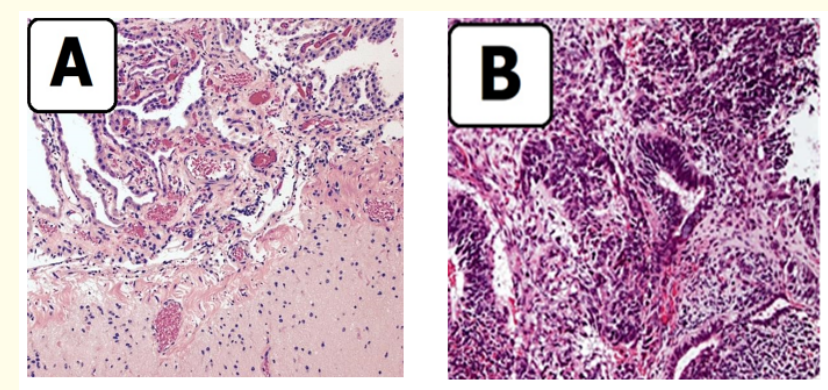

Figure 3 A,B: Histopathologic images revealed A, a mass composed of mature glial tissue, a wide range of cellular differentiation, a disorganized combination of mature adipose tissue, mucin secreting glands, tooth structure, skin adnexes, skeletal muscle, and bone. B, no immature component was present. There was no evidence of malignancy.

\section{Discussion}

The term teratoma comes from the Greek word "teraton" (meaning monster) and designates tumors arising from pluripotent cells [8]. These tumors are composed of multiple tissues derived from the three germ cell layers (ectoderm, endoderm, and mesoderm). Oral teratoma is one of the most frequent sites for head and neck teratomas, and do not present a clear gender predilection and may arise from anywhere in the oronasal cavity $[9,10]$. In one study authors found malignant teratoma to be more common in men in a ratio of 5:4 [11]. Oral teratomas have been classified into four subgroups: first, dermoid or hairy polyps that are the most common and contain only epidermal and mesodermal elements. Second, teratoids that consist of ectoderm, mesoderm, and endodermal elements but that are incompletely organized. Third, true teratomas that contain all three germ layers but with the greater histological organization and recognizable early organ differentiation. Fourth epignathus that are highly differentiated tumors with well-formed organs and limbs, which are rare and generally incompatible with life $[12,13]$. Another pathologic variation of epigraphic is fetus-in-fetus, which may be considered incomplete twinning of monozygotic twins at a primitive stage when axial development begins [14]. The most common theory supposes that an epignathus derives from pluripotential cells in Rathke's pouch that grow in a disorganized manner [15]. Many authors submitted that epignathi have been found to be associated with chromosomal abnormalities, such as duplication of $1 \mathrm{q}$ and 19p, ring X chromosome mosaicism, and the 49, XXXXY karyotype disorder $[16,17]$. Other studies have reported epignathi with no chromosomal abnormalities [18,19]. The parents in our case requested that we can not perform chromosome analysis, it was not available in our unit and too costly in the private sectors; accordingly, we were unable to establish a relationship between the tumor and the chromosomal assessments. Teratomas may be diagnosed antenatally by imaging, which permits early multidisciplinary management. In oral teratoma the most common symptoms are upper airway obstruction, dysphagia, and failure to gain weight [20]. Natal teeth are a distinctly rare condition defined as teeth present at birth, and neonatal teeth is which erupts within the first month of life [21]. The most common site of eruption is the lower central incisor area. The etiology of these teeth is still unknown, and careful evaluation of infants with natal or neonatal teeth is mandatory for possible syndromic conditions [22]. Our case was referred because of respiratory and feeding problems. Polyhydramnios and the severity of respiratory distress correlate with the size of the mass. Large oral teratoma typically emerges during the neonatal period in the head and neck is associated with high infant mortality rate in the absence of a well-prepared resuscitation team or meticulous delivery planning to secure the airway, as the majority of these teratomas are associated with obstruction of airway and difficulty in intubation [23]. The neonate's prognosis worsens as the size of the tumor increases. In the immature teratomas, tissues might arrange from embryonic to mature, which are scattered haphazardly throughout the tumor, which differ from the orderly organoid arrangement seen in a mature teratoma. Teratomas in the neonates are mostly benign and consist of mature tissue components [24]. While teratomas with malignant potential occur in adults and contain immature tissues, with a high incidence of malignancy. Incomplete resection and presence of primitive neural tissue are associated with a malignant relapse [20,23]. We must consider other clinical differential diagnoses of neonatal oral mass include embryonic congenital rhabdomyosarcoma, retinoblastoma, nasal glioma, heterotopic thyroid, cystic lymphangioma of the oral or nasopharyngeal regions, and sphenoid meningoencephalocele [10]. Assessment by the CT scan and Magnetic resonance imaging (MRI) play a key role in differentiating neonatal nasopharyngeal teratomas from other causes of neonatal neck mass [25]. Perinatal management of oral teratomas includes delivery by cesarean section and either resection operation on placental support (OOPS) or 
an Ex Utero intrapartum treatment (EXIT) procedure with or without tracheostomy. In centers that are more sophisticated, in utero fetoscopy management of fetuses with oral teratomas may be feasible in selected cases [26]. Unfortunately, we have neither such facilities nor experience in our pediatric surgery unit yet. Postnatal management of oral teratomas involves surgical resection, which is often curative, without recurrences if the initial surgery is successful.

\section{Conclusion}

Our case was extremely rare but important findings will assist our pediatric surgeons, pediatricians, and pathologists in developing future management strategies when they are enrolled or confronted with such cases. This case provides hope to all colleagues to improve the outcome through precise prenatal diagnosis, evaluation, and management roadmaps of potential cases of epignathus.

\section{Acknowledgements}

The author expresses sincere gratitude to all the pediatric surgery unit staff, pediatric dental outpatient's clinic, and all the colleagues in the radiology unit at the Maternity and Child Teaching Hospital, Al Qadisiya, Iraq, for their assistance.

\section{Conflict of Interest}

The authors declare that they have no competing interests.

\section{Bibliography}

1. Izadi K., et al. "A patient with an epignathus: management of a large oropharyngeal teratoma in a newborn". Journal of Craniofacial Surgery 14.4 (2003): 468-472.

2. Sandra M Halterman., et al. "Epignathus: Large Obstructive Teratoma Arising From the Palate". Cleft Palate-Craniofacial Journal 43.2 (2006): 244-246.

3. Smith NM., et al. "Oral teratoma (epignathus) with intracranial extension: a report of two cases". Prenatal Diagnosis 13.10 (1993): 945-952.

4. Kang KW., et al. "Prenatal ultrasonic diagnosis of epignathus". Journal of Clinical Ultrasound 6.5 (1978): 330-331.

5. Sherer DM., et al. "Prenatal 3-dimensional sonographic diagnosis of a massive fetal epignathus occluding the oral orifice and both nostrils at 35 weeks' gestation". Journal of Ultrasound in Medicine 25.11 (2006):1503-1505.
6. Ekici E., et al. "Prenatal diagnosis of epignathus causing acute polyhydramnios". Acta Obstetricia et Gynecologica Scandinavica 75.5 (1996): 498-501.

7. Vishal Khandelwal., et al. "Management of an infant having natal teeth". BMJ Case Reports (2013).

8. Kothari PR., et al. "Congenital naso-pharyngeal teratoma with cleft palate". Journal of Indian Association of Pediatric Surgeons 9 (2004): 42-45.

9. Cushing B., et al. "Germ cell tumors". In: Principles and practice of pediatric oncology. Fourth ed. Philadelphia: Lippincott Williams and Wilkins (2001).

10. Weaver RG., et al. "Teratomas of the head and neck". Surgical Forum 27.62 (1976): 539-544.

11. Laberge JM., et al. "Teratomas, dermoids, and other soft tissue tumors". In: Holocomb GWIII, Murphy JP, editors. Ashcraft's pediatric surgery. Fifth ed. Philadelphia, PA: Saunders Elsevier (2010): 915-935.

12. Kundra P and Krishnan S. "Airway management in children". Indian Journal of Anaesthesia 49 (2005): 300-307.

13. Ramani MN., et al. "Infant with a platal swelling an anaesthetic challenge". Indian Journal of Anaesthesia 46 (2002):217-218.

14. Clement K., et al. "Prenatal diagnosis of an epignathus: a case report and review of the literature". Ultrasound in Obstetrics and Gynecology 18.2 (2001): 178-181.

15. Daskalakis G., et al. "Prenatal diagnosis and management of fetal pharyngeal teratoma: a case report and review of the literature". Journal of Clinical Ultrasound 35.3 (2007): 159-163.

16. Raveh J. "Keystone" approach for intracranial nasofrontal dermoid sinuses". Plastic and Reconstructive Surgery 118 (2006): 271-272.

17. Cay A., et al. "Oral teratoma: a case report and literature review”. Pediatric Surgery International 20.4 (2004): 304-308.

18. Staboulidou I., et al. "Prenatal diagnosis of an epignathus associated with a 49, XXXXY karyotype--a case report". Fetal Diagnosis and Therapy 24 (2008): 313-317.

19. Dakpé S., et al. "Emergency management of a congenital teratoma of the oral cavity at birth and three-year follow-up". International Journal of Oral and Maxillofacial Surgery 43.4 (2014): 433-436. 
20. Wang AC., et al. "Congenital giant epignathus with intracranial extension in a fetal". Chinese Medical Journal 130.19 (2017): 2386-2387.

21. Mehmet Nevzat Cizmeci., et al. "Neonatal tooth in a preterm infant”. European Journal of Pediatrics 172.2 (2013): 279.

22. Basavanthappa NN., et al. "Natal and neonatal teeth: A retrospective study of 15 cases". European Journal of Dentistry 5.2 (2011):168-172.

23. Becker S., et al. "A congenital teratoma with a cleft palate: report of a case". British Journal of Oral and Maxillofacial Surgery 45.4 (2007): 326-327.

24. Takeuchi K., et al. "Prenatal evaluation of bidirectional epignathus: comparison of ultrasonography and MRI". Fetal Diagnosis and Therapy 18.1 (2003): 26-28.

25. Chiu HH., et al. "The EXIT (ex utero intrapartum treatment) procedure". Journal of the Formosan Medical Association 107.9 (2008): 745-748.

26. Skarsgard ED., et al. "The OOPS procedure (operation on placental support): in utero airway management of the fetus with prenatally diagnosed tracheal obstruction". Journal of Pediatric Surgery 31.6 (1996): 826-828.

Volume 2 Issue 10 October 2019

(C) All rights are reserved by Mohammed J Aboud. 